\title{
Distribution of NDMI Carbapenemase-Producing Proteeae Strains on High-Risk Hospital Wards
}

This article was published in the following Dove Press journal:

Infection and Drug Resistance

\author{
Maria Rus (D) ${ }^{1,2}$ \\ Monica Licker $\mathbb{D}^{1-3}$ \\ Corina Musuroi ${ }^{2}$ \\ Edward Seclaman ${ }^{4}$ \\ Delia Muntean (iD) ${ }^{1-3}$ \\ Natalia Cirlea ${ }^{2}$ \\ Alina Tamas ${ }^{2}$ \\ Silvana Vulpie ${ }^{2}$ \\ Florin George Horhat $\mathbb{D}^{1,3}$ \\ Luminita Baditoiu (D) ${ }^{3,5}$ \\ 'Department of Microbiology, "Victor \\ Babes" University of Medicine and \\ Pharmacy, Timisoara, Romania; ${ }^{2 " P i u s}$ \\ Brinzeu" County Clinical Emergency \\ Hospital, Timisoara, Romania; \\ ${ }^{3}$ Multidisciplinary Research Center on \\ Antimicrobial Resistance, "Victor Babes" \\ University of Medicine and Pharmacy, \\ Timisoara, Romania; ${ }^{4}$ Department of \\ Biochemistry, "Victor Babeș" University \\ of Medicine and Pharmacy, Timișoara, \\ Romania; ${ }^{5}$ Department of Epidemiology, \\ "Victor Babeș" University of Medicine \\ and Pharmacy, Timișoara, Romania
}

\begin{abstract}
Background: Carbapenem-resistant Proteeae (CRP) is a group of multidrug-resistant (MDR) microorganisms that raise special treatment problems due to their intrinsic resistance to colistin. In this study, our aim is to provide a phenotypic and molecular characterization of the carbapenemases secreted by CRP strains isolated from inpatients from an intensive care unit (ICU) and surgical wards, as well as the identification of the risk factors involved in their acquisition.
\end{abstract}

Methods: An observational, cross-sectional study was performed which included all Proteeae strains isolated in samples from inpatients on high-risk wards of the largest university hospital in Western Romania, from July 2017 to April 2019. Meropenemresistant strains $(\mathrm{N}=65)$ with $\mathrm{MIC} \geq 16 \mu \mathrm{g} / \mathrm{mL}$ were subjected to a singleplex PCR assay for the detection of blaNDM, blaVIM and blaCTX-M genes. The analysis of risk factors was performed by logistic regression.

Results: Out of 8317 samples that were processed, 400 Proteeae strains were isolated: $64 \%$ belonging to the genus Proteus, 26.75\% to the genus Providencia and $9.25 \%$ to the genus Morganella. Most CRP strains (N=56) were of MBL type, and 55 had the blaNDM gene as the prevalent gene substrate. P. stuartii was the main species that provided the circulating MDR strains. Most CRP strains came from patients admitted to ICU, being isolated mainly from bronchial aspirates and blood cultures. Multivariate analysis revealed 3 independent risk factors - mechanical ventilation $>96$ h (HR: 40.51 [13.65-120.25], $\mathrm{p}<0.001)$, tracheostomy (HR: 2.65 [1.14-6.17], $\mathrm{p}=0.024)$ and prolonged antibiotic therapy (HR: 1.01 [1.00-$1.02], \mathrm{p}=0.03)$.

Conclusion: There is a significant increase in the incidence of CR P. stuartii strains, the MBL-blaNDM type being predominant. These strains presented various other resistance mechanisms, being often extremely difficult to treat and led to an excess of lethality of $27.16 \%$.

Keywords: carbapenem-resistant Proteeae, intensive care unit, $P$. stuartii

\section{Introduction}

The Proteeae tribe includes the following genera: Proteus, Morganella and Providencia. These are opportunistic microorganisms belonging to the Morganellaceae family, order Enterobacterales ${ }^{1,2}$ that represent a particular challenge because of their intrinsic resistance to tigecycline, colistin, nitrofurantoin and reduced intrinsic sensitivity to imipenem. These limitations make the antimicrobial therapy for infections caused by these microorganisms a particular challenge from the start. The most sensitive genus is Proteus, followed by Morganella and Providencia. $^{3,4}$
Correspondence: Monica Licker Department of Microbiology, "Victor Romania

Tel +40 0256-707513

Email licker.monica@umft.ro

Corina Musuroi

Pius Brinzeu" County Clinical Emergency Hospital, No. 156 L.Rebreanu, Timisoara 300723, Romania

Tel +40745050232

Email cormusI@yahoo.com
Infection and Drug Resistance 2020:13 475|-476| 
On this background of natural resistance, Proteeae have developed the phenomenon of acquired resistance and even multi-resistance, so that in 2017 the World Health Organization included extended spectrum betalactamase-producing (ESBL) and carbapenem-resistant enterobacteria (CRE) (including Proteus spp., Providencia spp. and Morganella spp.) in the first category of MDR pathogens, having Priority 1: CRITICAL. ${ }^{5}$

The enzymes responsible for Proteeae resistance to carbapenems, which is considered to be of high epidemiological and clinical significance, are KPC, OXA-48 and NDM, VIM and IMP metallo- $\beta$-lactamases (MBL). ${ }^{6-13}$

KPC is active on the classes of penicillins, cephalosporins, $\beta$-lactamase inhibitors and also on carbapenems and aztreonam, its activity being moderately inhibited by clavulanic acid. $^{3}$

MBLs have a significant hydrolytic activity on carbapenems, but without any effect on aztreonam, which they cannot hydrolyze. ${ }^{14}$ The association of MBL and ESBL secretion is common in MDRs isolated in samples from immunosuppressed patients.

OXA-48 has a specific spectrum of activity, hydrolyzing penicillins and carbapenems, but without any effect on cephalosporins and aztreonam. In addition, its activity on carbapenems is not as strong as that of KPCs and MBLs, which leads to the difficultly of detection by phenotypic tests. $^{3,14}$

The genetic material responsible for enzyme secretion is frequently identified at the extrachromosomal level, on plasmids, which represent the transfer vector of the gene substrate for the MDR behaviour, especially in inpatients. They may have representatives of the same species as the target, or can be transferred, even between different species. This transfer is especially likely to occur on high-risk wards, being helped by the patient's suppressed immune system, as well as by the large number of invasive therapeutic procedures performed. This creates conditions suitable for the occurrence of infection outbreaks with MDR microorganisms belonging to the species known for this behavior, even with less frequently identified strains. ${ }^{3,4,14}$

Such a situation has been reported in several Romanian hospitals over the last decade, where there has been an alarming increase in the incidence of carbapenem-resistant Proteeae (CRP) in samples from patients admitted to highrisk wards. ${ }^{15-17}$

In this context, the present study aimed to provide a phenotypic and molecular characterisation of carbapenemases secreted by Proteeae strains isolated from patients admitted to high-risk wards in a university hospital in Western Romania and also to identify the associated factors involved in the acquisition of these strains.

\section{Materials and Methods Study Design and Sampling}

This observational, cross-sectional study included all strains from the Proteeae group isolated from samples of ICU and surgical ward inpatients from "Pius Brinzeu" County Clinical Emergency Hospital Timișoara (SCJUPBT), from July 2017 to April 2019. This institution is a tertiary medical care unit with 1174 beds, of which 32 beds are in ICU (with mixed pathology, medical and surgical), and with 501 beds on surgical wards (urology, orthopedics, general surgery, neurosurgery, vascular surgery, plastic surgery).

From the total of 400 strains identified, 65 meropenemresistant strains (MIC $\geq 16 \mu \mathrm{g} / \mathrm{mL}$ ) were collected to assess the presence of blaNDM, blaVIM genes, and for those 43, out of the 65 , which had associated cefepime resistance $(\mathrm{MIC} \geq 64 \mu \mathrm{g}) / \mathrm{mL}$ ) we also assessed the presence of blaCTX-M genes.

Patients with an ICU admission of less than 1 hour, as well as those under 18 years of age, were excluded. Those discharged and then readmitted to the same ward but on a different date were included in the database only if a new species of Proteeae was isolated or a new resistance phenotype was identified, completely different from the one previously included.

Demographic and medical data for the patients included in this study were collected (diagnosis on admission, date of admission, length of hospital stay, discharge status, invasive therapeutic procedures and medical conditions that may increase host's vulnerability). Bacteriology diagnostic data and antimicrobial sensitivity were also taken into account. For those 65 strains subjected to molecular analysis, data regarding the type of carbapenemase and its gene substrate were collected.

Data collection was performed with the approval of the Ethics Commission of SCJUPBT (no. 149/6.02.2019), according to the policy covering patient data protection and patient confidentiality.

\section{Microbiological Method}

To avoid duplication and phenotypic changes induced by antibiotic selection pressure, only the first strain, isolated from various samples of the same patient, was included. 
As a result, 400 strains from the Proteeae group having clinical significance were identified and studied, being isolated from samples such as bronchial aspirates, blood cultures, wound swabs, urine cultures, catheter tips, purulent secretions, peritoneal fluid, sputum cultures, biliary fluid, ascites fluid, skin fragments, genital specimens and auricular secretions.

The primary culture was performed according to the working protocol of the Bacteriology Laboratory of SCJUPBT. The identification and antimicrobial sensitivity testing were performed using the automatic system Vitek 2 Compact (BioMérieux, France), according to CLSI. ${ }^{18}$ The reference strains used were Escherichia coli ATCC 25922 and Klebsiella pneumoniae ATCC 1705.

The identification of microbial mechanisms for carbapenem resistance with the detection of $\mathrm{KPC}, \mathrm{MBL}$ and OXA-48 carbapenemases secreted by Proteeae strains was performed using the KPC/MBL and OXA-48 kit (Rosco Diagnostica, Taastrup, Denmark).

MDR strains have been defined as resistant to at least one antibacterial agent from three or more classes of antibiotics. $^{19}$

The monthly percentage of multidrug resistance was calculated by comparing the number of MDR strains from the same tribe/species to the total number of strains belonging with that tribe/species, isolated over that month.

The monthly share of one species in the total number of Proteeae strains was calculated by comparing the number of strains of that species, isolated over that month, with the total number of Proteeae strains isolated over the same month.

\section{Molecular Method}

All CRPs were grown for $24 \mathrm{~h}$ at $37^{\circ} \mathrm{C}$ in broth medium. Bacterial DNA was extracted with PureLink Microbiome DNA Purification kit (Invitrogen) according to the manufacturer's protocol, starting from a $5 \mathrm{~mL}$ sample. DNA concentration was assessed with NanoDrop ND1000. All PCRs were performed with AmpliTaq Gold 360 Master Mix, in $0.2 \mathrm{~mL}$ thin-walled PCR tubes, with $25 \mu \mathrm{L}$ reactions mix, on a Veriti 96-Well Thermal Cycler, using $10 \mathrm{ng}$ of genomic DNA (Thermo Fisher Scientific).

The isolates were analyzed for carbapenemase identification using a singleplex PCR assay, according to the method described by Poirel et al. ${ }^{20}$ Sets of primers for blaNDM and blaVIM genes were included. For the analysis of ESBL strains, the method described by Akhi et al for the blaCTX-M primer pair was used. ${ }^{21}$
The following reference strains were used for positive control: Escherichia coli R45 (NDM), R61 (VIM) and R1818 (CTX-M) purchased from the Université de Fribourg. For negative control, Escherichia coli ATCC 25922 was used.

\section{Statistical Analysis}

Data analysis was performed using IBM SPSS Statistics 20 (SPSS Inc., Chicago, IL). Continuous variables were characterized by mean values and the interval between quartiles (IQR) and the category type was characterized by value and percentage. The $95 \%$ confidence interval was calculated for all variables. Data distribution testing was performed using the Kolmogorov-Smirnov test. The numerical variables were compared with the $t$-test for independent samples (for those with Gaussian distribution), with the Mann-Whitney $U$-test for those that do not have a normal distribution, and with the chi-squared test (Fisher exact test) for the nominal ones. The bivariate correlation was achieved by applying the Pearson correlation. The variables with $\mathrm{p}<0,05$ were investigated by logistic regression, choosing the model according to the Nagelkerke $\mathrm{R}^{2}$ coefficient and the test for assessing the deviation from the theoretical model of Hosmer and Lemeshow. All statistical tests were calculated with two extremities and the threshold of statistical significance for $\mathrm{p}$ was considered 0.05 .

\section{Results}

From July 2017 to April 2019, 8317 samples collected from ICU and surgical wards were processed. Their distribution is presented in Table 1 .

From these samples, 400 strains from the Proteeae tribe were identified and studied. Two hundred and fiftysix $(64.00 \%)$ of these strains belonged to the genus Proteus (P. mirabilis, P. vulgaris, P. penneri), 107 $(26.75 \%)$ to the genus Providencia (P. stuartii, $P$. rettgeri), the least represented being the genus Morganella (M. morganii, M.sibonii), with 37 (9.25\%) isolated strains (Table 2).

Most Proteeae strains came from wound swabs (39.50\%, 95\% CI: 34.7-44.5), bronchial aspirates $(20 \%$, 95\% CI: 16.3-24.3), urine cultures (15.25\%, 95\% CI: 11.9-19.2), blood cultures (8.5\%, 95\% CI: 6.0-11.8) and catheter tips (7\%, 95\% CI: 4.8 to 10.1) (Figure 1).

From these samples, $49.75 \%$ (95\% CI: 44.8-54.8) of the Proteeae strains came from patients admitted to ICU and the remaining 50.25\% (95\% CI: 45.2-55.2) came from those admitted to surgical wards. 
Table I Distribution of Samples by Clinical Wards

\begin{tabular}{|c|c|c|c|c|c|c|c|c|c|c|c|c|c|c|c|}
\hline \multirow[t]{2}{*}{ Department } & \multirow[t]{2}{*}{ Total } & \multicolumn{2}{|c|}{$\begin{array}{l}\text { Bronchial } \\
\text { Aspirates }\end{array}$} & \multicolumn{2}{|c|}{ Sputum } & \multicolumn{2}{|c|}{$\begin{array}{l}\text { Blood } \\
\text { Cultures }\end{array}$} & \multicolumn{2}{|c|}{$\begin{array}{l}\text { Catheter } \\
\text { Tips }\end{array}$} & \multicolumn{2}{|c|}{$\begin{array}{l}\text { Urine } \\
\text { Cultures }\end{array}$} & \multicolumn{2}{|c|}{$\begin{array}{l}\text { Wound } \\
\text { Swabs }\end{array}$} & \multicolumn{2}{|c|}{$\begin{array}{l}\text { Other } \\
\text { Samples }\end{array}$} \\
\hline & & $\mathbf{N}$ & $\%$ & $\mathbf{N}$ & $\%$ & $\mathbf{N}$ & $\%$ & $\mathbf{N}$ & $\%$ & $\mathbf{N}$ & $\%$ & $\mathbf{N}$ & $\%$ & $\mathbf{N}$ & $\%$ \\
\hline ICU & 3831 & 1513 & 39.49 & 57 & $\mathrm{I} .48$ & 1192 & 31.11 & 303 & 7.9 & 209 & 5.45 & 353 & 9.21 & 204 & 5.33 \\
\hline General surgery & 781 & 0 & 0 & 10 & 1.28 & 21 & 2.68 & 12 & 1.54 & 33 & 4.23 & 466 & 59.67 & 239 & 30.6 \\
\hline Neurosurgery & 217 & 14 & 6.45 & 0 & 0 & 20 & 9.22 & 10 & 4.61 & 39 & 17.97 & 69 & 31.79 & 65 & 29.95 \\
\hline Vascular surgery & 925 & 1 & 0.11 & 0 & 0 & 5 & 0.54 & 4 & 0.43 & 12 & 1.3 & 894 & 96.65 & 9 & 0.97 \\
\hline Plastic surgery & 824 & 3 & 0.36 & 1 & 0.12 & II & 1.33 & 6 & 0.73 & 12 & 1.46 & 787 & 95.51 & 4 & 0.49 \\
\hline Urology & 1095 & 0 & 0 & 5 & 0.45 & 31 & 2.83 & 8 & 0.73 & 991 & 90.5 & 43 & 3.92 & 17 & 1.55 \\
\hline Orthopaedics & 497 & 5 & I & 1 & 0.201 & 13 & 2.61 & 3 & 0.6 & 76 & 15.29 & 375 & 75.45 & 24 & 4.83 \\
\hline Polytrauma & 147 & 5 & 3.4 & 0 & 0 & 15 & 10.2 & 19 & 12.93 & 19 & 12.93 & 82 & 55.78 & 7 & 4.76 \\
\hline Total & 8317 & $|54|$ & 18.53 & 74 & 0.89 & 1308 & 15.73 & 365 & 4.39 & $139 \mid$ & 16.72 & 3069 & 36.9 & 569 & 6.84 \\
\hline
\end{tabular}

Table 2 Phenotypic and Molecular Profile of Carbapenem-Resistant Proteeae Strains

\begin{tabular}{|c|c|c|c|c|c|c|c|c|c|c|}
\hline \multirow[t]{2}{*}{ Species } & \multirow[t]{2}{*}{$N=400$} & \multirow[t]{2}{*}{ KPC } & \multirow{2}{*}{$\begin{array}{l}\text { OXA- } \\
48\end{array}$} & \multicolumn{3}{|l|}{ MBL } & \multirow{2}{*}{$\begin{array}{l}\text { blaCTX- } \\
\text { M }\end{array}$} & \multirow{2}{*}{$\begin{array}{l}\text { blaCTX- } \\
\text { M +KPC }\end{array}$} & \multirow{2}{*}{$\begin{array}{l}\text { blaCTX- } \\
\text { M } \\
+ \text { blaNDM }\end{array}$} & \multirow{2}{*}{$\begin{array}{l}\text { Molecularly } \\
\text { Analyzed } \\
\text { Strains }\end{array}$} \\
\hline & & & & blaNDM & blaVIM & $\begin{array}{l}\text { blaNDM } \\
+ \text { blaVIM }\end{array}$ & & & & \\
\hline P. mirabilis [n, \%] & $245(61.25)$ & I $(6.25)^{*}$ & $0(0)$ & $12(75.00)$ & I (6.25) & $0(0)$ & I (6.25) & $0(0)$ & I (6.25) & $16(100)$ \\
\hline Proteus vulgaris [n, \%] & $10(2.50)$ & I & I & I & 1 & 1 & 1 & I & 1 & $0(0)$ \\
\hline Proteus penneri $[\mathrm{n}, \%]$ & I $(0.25)$ & I & I & I & I & I & I & I & I & $0(0)$ \\
\hline P. rettgeri $[\mathrm{n}, \%]$ & $13(3.25)$ & $0(0)^{*}$ & $0(0)$ & $I(100)$ & $0(0)$ & $0(0)$ & $0(0)$ & $0(0)$ & $0(0)$ & $I(100)$ \\
\hline P. stuartii [n, \%] & $94(23.50)$ & $3(6.25)^{*}$ & $2(4.17)^{*}$ & 37 (77.08) & $0(0)$ & I (2.08) & I (2.08) & I (2.08) & $3(6.25)$ & $48(100)$ \\
\hline $\begin{array}{l}\text { Morganella morganii } \\
{[\mathrm{n}, \%]}\end{array}$ & $35(8.75)$ & I & 1 & I & I & 1 & I & I & I & $0(0)$ \\
\hline $\begin{array}{l}\text { Morganella sibonii } \\
{[\mathrm{n}, \%]}\end{array}$ & $2(0.50)$ & I & l & I & 1 & I & l & l & l & $0(0)$ \\
\hline Total $[\mathrm{n}, \%]$ & $400(100)$ & $4(6.15)^{*}$ & $2(3.08)$ & $50(76.92)$ & I (1.54) & I (1.54) & $2(3.08)$ & I (1.54) & $4(6.15)$ & $65(100)$ \\
\hline
\end{tabular}

Note: *Percentages reported to the total number of strains, of the same species, molecularly analyzed.

Monthly percentage(s) of multidrug resistance of Proteeae strains correlated strongly positive with P.stuartii monthly share series, from the total Proteeae $(r=0.833, \mathrm{p}<0.001)$ and strongly negative, with the series of $P$. mirabilis monthly share $(r=-0.713, \mathrm{p}<0.001)$. The percentage of $69.39 \%$ of Proteeae multiresistance variability can be explained by the P. stuartii percentage variability in the total of Proteeae strains per month $\left(r^{2}=0.6938\right)$ (Figure 2).

Among these 400 Proteeae strains, 65 CRP strains were identified (meropenem MIC $\geq 16 \mu \mathrm{g} / \mathrm{mL}$ ), which were subjected to genotyping in order to detect the presence of the blaNDM, blaVIM genes. The incidence of CRP strains was $16.25 \%, 6.25 \%$ for the genus Proteus and $45.79 \%$ for the genus Providencia.

Most of the CRP strains were isolated from patients admitted to ICU $-86.15 \%$ (95\% CI: $19.9-43.4)$, most of them $(63 \%)$ in bronchial aspirates $(32.31 \%, 95 \%$ CI: 21.2 $-45.1)$ and blood cultures (30.7\%, 95\% CI: 19.9-43.4).
The comparison of the total Proteeae group with the subgroup of CRP strains reveals that CR strains were more frequently isolated from bronchial aspirates and blood cultures and less frequently from wound swabs, indicating their more frequent involvement in lower respiratory tract infections and sepsis and less so in surgical wound infections (Table 3).

From a phenotypical point of view, $100 \%$ of CRP strains were resistant to imipenem, meropenem, piperacillin, ceftazidime and $95.38 \%$ to piperacillin-tazobactam (95\% CI: 87.1-99.0). Resistance to carbapenems was associated with resistance to aminoglycosides: gentamicin (84.62\%, 95\% CI: 73.5-92.4), amikacin (55.38\%, 95\% CI: 42.5-67.7), fluoroquinolones: ciprofloxacin $(95.38 \%$, 95\% CI: 87.1-99.0), levofloxacin (86.15\%, 95\% CI: 75.3-93.5), and trimethoprim-sulfamethoxazole (98.46\%, 95\% CI: 91.7-100,0), having major negative effects on treatment options available for these patients (Table 4). 


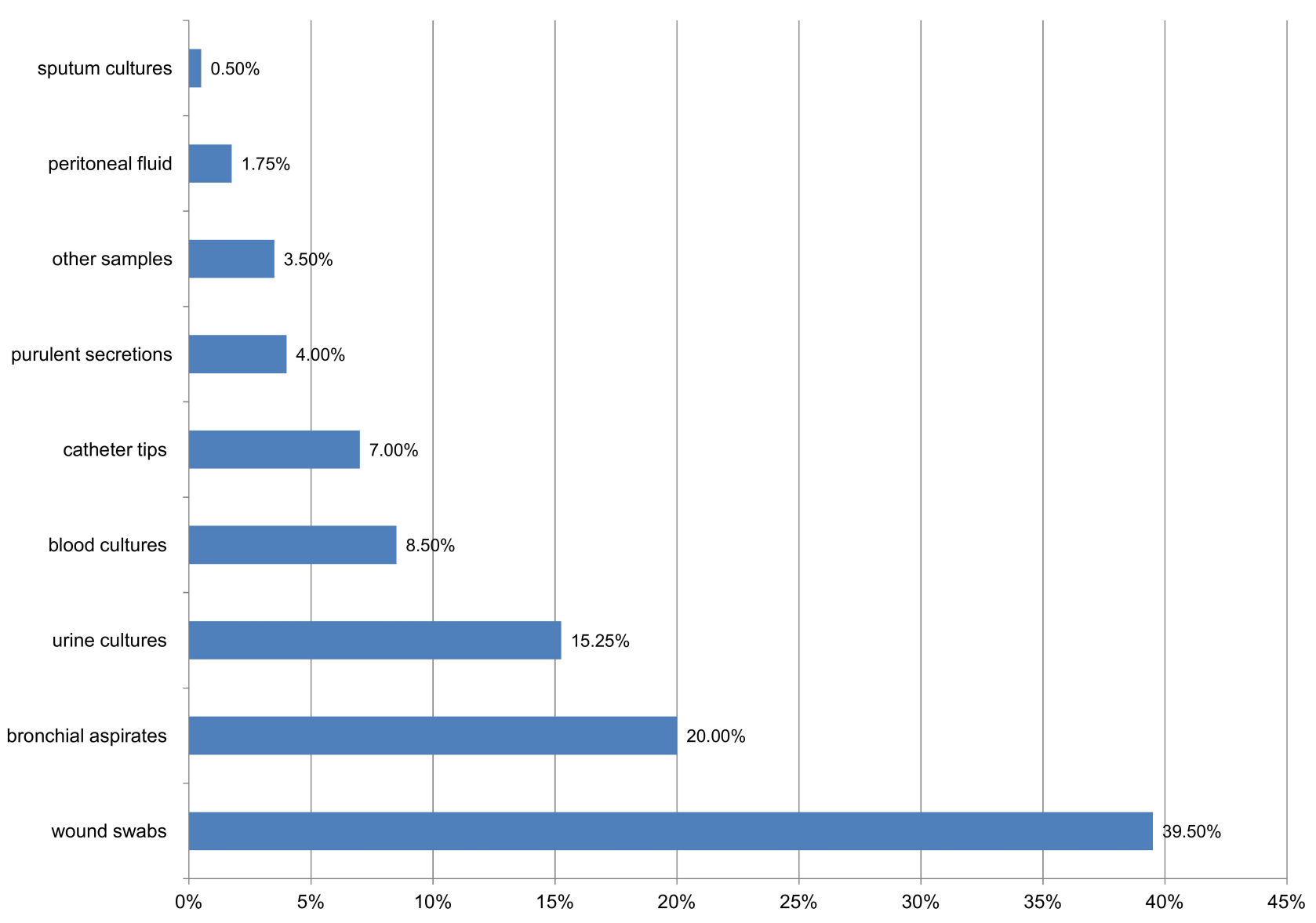

Figure I Distribution of Proteeae strains in samples.

Regarding the behaviour of fourth-generation cephalosporins, $66.15 \%$ of the strains were resistant to cefepime (95\% CI: 53.4-77.4), 7 of Proteae strains having blaCTX$\mathrm{M}$ gene identified by PCR test $(10.76 \% ; 95 \%$ CI: 4.4-20.9).

The identification of the bacterial resistance mechanism for carbapenems by Rosco Diagnostica test detecting KPC, MBL and OXA-48 carbapenemases secreted by Proteeae strains, and of gene substrate for antibiotic resistance identifying blaNDM and blaVIM genes, highlighted the CRP profile shown in Table 2.

It should be noted that the most frequently represented was MBL carbapenemase, produced by 56 of the CRP strains (86.15\%; 95\% CI: 75.3-93.5), having as prevalent gene substrate, in 55 strains, the blaNDM gene $(84.62 \%$; 95\% CI: 73.5-92.4). Most of the CRP strains belonged to the P. stuartii species, the most prevalent being the MBLblaNDM strains (61.54\% of the total CRP strains), followed by the $P$. mirabilis strains of MBL-NDM type (20\% of the total CRP strains). It is noteworthy that an association of blaNDM + bla VIM genes was identified in one of the $P$. stuartii strains. In two strains, one of $P$. mirabilis and the other of P.stuartii, although phenotypically resistant to carbapenems, no carbapenemase synthesis was identified, but they showed the blaCTX-M gene.

For the analysis of the predictive factors for the acquisition of CRP strains, the subgroup of CR strains was compared with the 222 Proteeae strains that did not meet the MDR criteria (included in the total of 400 strains that were analyzed). The results are presented in Table 5.

Moreover, the lethality of cases with CRP strains was $49.23 \%$ versus $33.43 \%$ in the total sample $(\mathrm{p}=0.014)$ and $22.07 \%$ among those with carbapenem-sensitive Proteeae strains $(\mathrm{p}<0.001)$.

\section{Discussion}

The current study aimed to analyse the behaviour of Proteeae strains regarding the frequency of occurrence, distribution in different samples, antimicrobial resistance profile and phenotypic/molecular characterization of CR strains, as well as identifying the risk factors involved. 


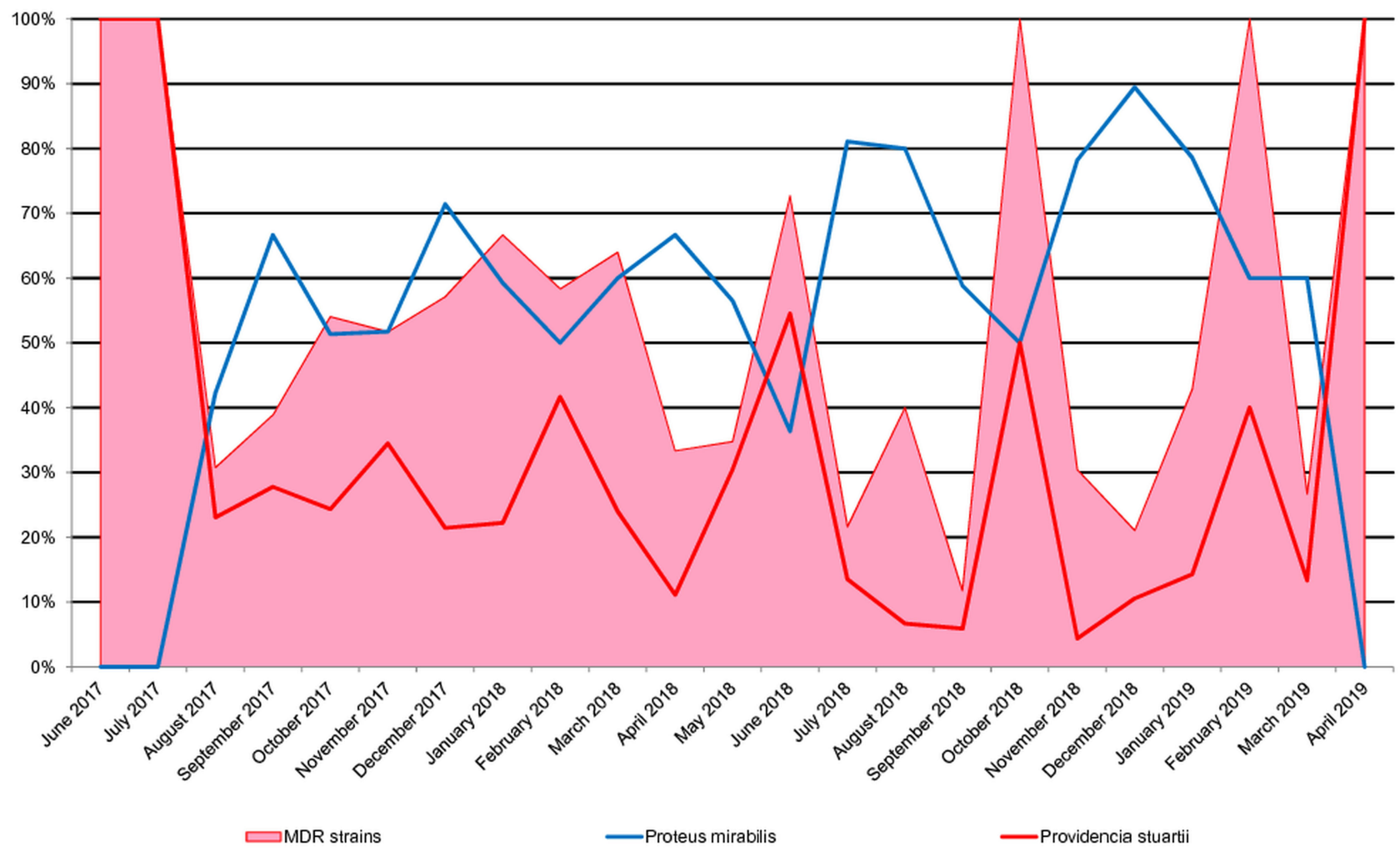

Figure 2 Evolution of the multidrug resistance of Proteeae strains per month in parallel with the monthly share of the two species.

The incidence of Proteeae strains among the total number of samples in the study was $4.81 \%$ represented mainly by P. mirabilis and P. stuartii, with a percentage of $2.94 \%$ and $1.13 \%$, respectively and a ratio of $2.6: 1$. Compared with a study conducted between January 2012 and December 2013 in the ICU of the same hospital ${ }^{17}$ a significant decrease can be observed in the ratio between these two strains in the last 5 years, due to a reduction in the percentage of $P$. mirabilis and the continuance of a relatively constant incidence of $P$. stuartii strains (the former ratio being $6: 1)$.

We noticed a balanced distribution of the total group of Proteeae strains $(48.25 \%, 50.25 \%)$ and the subgroup of

Table 3 Comparative Analysis of the Origin of Proteeae Strains*

\begin{tabular}{|l|l|l|l|l|l|}
\hline \multirow{2}{*}{ Sample } & \multicolumn{3}{|l|}{$\begin{array}{l}\text { Subgroup of } \\
\text { CRP Strains }\end{array}$} & $\begin{array}{l}\text { Total Group of } \\
\text { Proteeae } \\
\text { Strains }\end{array}$ & P \\
& \multicolumn{2}{|c|}{} & \multicolumn{1}{|l|}{} \\
\cline { 2 - 5 } & $\mathbf{n = 6 5}$ & $\%$ & $\mathbf{N}=400$ & $\%$ & \\
\hline Bronchial aspirates & 21 & 32.31 & 80 & 20.00 & 0.025 \\
Blood cultures & 20 & 30.77 & 34 & 8.50 & $<0.001$ \\
Wound swabs & 7 & 10.77 & 158 & 39.50 & $<0.001$ \\
\hline
\end{tabular}

Note: *For the rest of the samples, there were no statistically significant differences.
P. mirabilis strains $(44.89 \%, 55.11 \%)$ in the ICU and on surgical wards, while $P$. stuartii strains were identified mostly in the ICU (77.65\%), where the patients are immunocompromised. Proteeae strains were mainly isolated in wound swabs and bronchial aspirates.

Antimicrobial testing has shown an increased frequency of MDR Proteeae strains (44.50\%) and a correlation of multidrug resistance with the share of P. stuartii strains, which thus proves to be responsible for the major therapeutic difficulties in patients admitted to the ICU.

A special group of MDR strains was represented by CRP, the P. mirabilis - CR representing $4 \%$ of the total Proteeae and P.stuartii- CR12\%, with a ratio of $3: 1$ between strains. What can be observed here is a reversal of the ratio between the two species for the CR subgroups and a dominance of the P. stuartii, which was the main species of Proteeae that provided the circulating MDR strains.

Regarding the phenotypical resistance to other classes of antimicrobials, most CRP strains have associated resistance to fluoroquinolones and trimethoprimsulfamethoxazole and reduced susceptibility to aminoglycosides, especially to gentamicin. Moreover, CR Proteeae were 


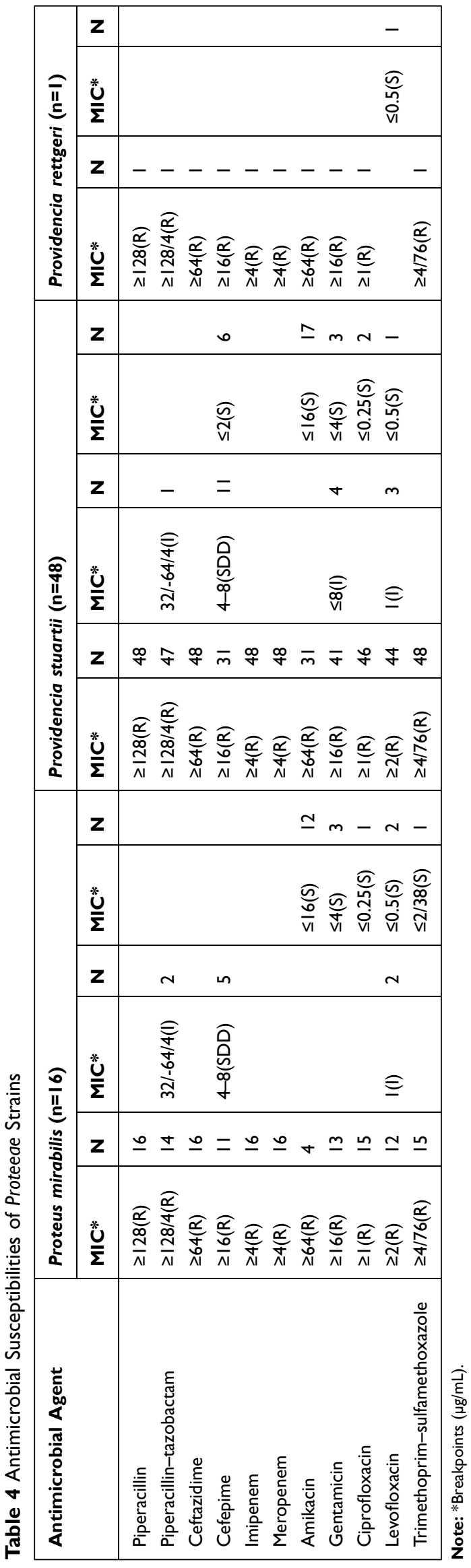

resistant to fourth-generation cephalosporins (Cefepime) in a proportion of $66.15 \%$.

Most CRP strains $(84.15 \%)$ were isolated in the samples of ICU patients, with a numerical accumulation in blood cultures and bronchial aspirates, significantly higher than in the case of the total group of Proteeae strains ( $\mathrm{p}<0.001, \mathrm{p}=0.025$ ).

Among the carbapenemases that were identified, the best represented molecular class was that of MBLs, produced by $86.15 \%$ of CRP strains, $84.62 \%(n=55)$ being of NDM type and only two strains of VIM type. KPC and OXA-48 carbapenemases were also identified, but less frequently. However, it was noted that all 5 KPC carbapenemase-producing strains came from neurosurgery, and the two OXA-48 strains were isolated on general surgery and neurosurgery wards.

P.stuartii- CR strains, representing the majority of the CRP group, were confirmed as producing MBL-NDM in $85.42 \%$ of cases, and less frequently KPC, OXA-48 and VIM $(n=4, n=2, n=0)$. Only one strain showed a combination of blaNDM and blaVIM. The finding correlates with the data from the literature showing that the most common carbapenemase identified in this species was of MBL type, predominantly NDM- $1^{22-28}$ and only in isolated cases MBL-VIM- ${ }^{29-31}$ or MBL-VIM-19. ${ }^{32}$ Moreover, only one study conducted in Mexico in 2015 identified a strain of $P$. stuartii secreting two carbapenemases, blaNDM - blaIMP. $^{33}$

Regarding the P. mirabilis - CR strains, most of them were identified as MBL-NDM type $(n=13)$, one MBLVIM and one KPC type. These strains did not show a gene substrate for OXA-48 synthesis. However, studies have shown that $P$. mirabilis - CR strains are versatile in terms of carbapenemase production, MBL-NDM, VIMand IMP, ${ }^{8,34-36}$ or KPC-producing strains being frequently reported. ${ }^{6,7,9}$

With regards to the $P$. rettgeri strain, this was confirmed to be MBL-NDM type. In general, these strains are rarely described in the literature and there are only a few studies conducted on larger groups. In 2005, Shiroto identified 9 strains of CR P. retgerii, out of a total of 495 Proteeae (1.81\%) and 8 of these strains had the blaIMP-1 gene. ${ }^{8}$ Jui-Hsuan Yang (2020) stated that 9 CR strains were identified out of a total of 142 Proteeae $(6.33 \%)$, from 2008 to $2011 .{ }^{37}$ In 2017, Mariappan et al identified two strains (NDM type) of P. rettgeri in a batch of 111 Enterobacteriaceae. $^{38}$ 
Table 5 Analysis of Associated Factors Involved in Infections with CR Proteeae Strains

\begin{tabular}{|c|c|c|c|c|c|c|}
\hline Variables & $\begin{array}{l}\text { CRP Group } \\
\mathrm{nI}=65\end{array}$ & $\begin{array}{l}\text { P-S Group } \\
\text { n2=222 }\end{array}$ & $\mathbf{p}$ & OR $[95 \% \mathrm{Cl}]$ & $\mathbf{p}$ & HR $[95 \% \mathrm{Cl}]$ \\
\hline Age [median, IQR] & $60[50.00-68.00]$ & $64.00[54.00-72.00]$ & 0.005 & $0.975[0.96-0.99]$ & & \\
\hline Days of hospital stay [median, IQR] & $45.00[32.00-69.00]$ & $23.00[8.00-53.00]$ & $<0.001$ & $1.00[1.00-1.01]$ & & \\
\hline Days of antibiotherapy [median, IQR] & $35.00[16.00-52.00]$ & $9.00[4.00-24.00]$ & $<0.001$ & $1.01[1.00-1.02]$ & 0.033 & $1.01[1.00-1.02]$ \\
\hline Number of antibiotics [median, IQR] & $5.00[4.00-7.00]$ & $2.00[1.00-3.00]$ & $<0.001$ & $1.57[1.38-1.79]$ & & \\
\hline Days of CVC [median, IQR] & $28.00[16.00-48.00]$ & $2.00[0.00-12.00]$ & $<0.001$ & $1.03[1.02-1.04]$ & & \\
\hline Days of urinary catheter [median, IQR] & $31.00[20.00-55.00]$ & $0.00[0.00-12.00]$ & $<0.001$ & $1.03[1.02-1.04]$ & & \\
\hline Female $[\mathrm{n}, \%]$ & $21(32.31)$ & $82(36.94)$ & 0.493 & $0.81[0.42-1.52]$ & & \\
\hline Male $[\mathrm{n}, \%]$ & $44(67.69)$ & $140(63.04)$ & & & & \\
\hline \multirow{2}{*}{$\begin{array}{l}\text { Previous antibiotherapy } \\
\text { Yes/No [n, \%] }\end{array}$} & $22(33.85)$ & $28(|2.6|)$ & \multirow[t]{2}{*}{$<0.001$} & \multirow[t]{2}{*}{$3.54[1.76-7.13]$} & & \\
\hline & $43(66.15)$ & $194(87.39)$ & & & & \\
\hline \multirow{2}{*}{$\begin{array}{l}\text { Mechanical ventilation } \\
\text { Yes/No [n, \%] }\end{array}$} & 61 (93.85) & $56(25.23)$ & \multirow[t]{2}{*}{$<0.001$} & \multirow[t]{2}{*}{$45.21[15.5|-| 76.26]$} & & \\
\hline & $4(6.15)$ & $166(77.77)$ & & & & \\
\hline $\begin{array}{l}\text { Length of mechanical ventilation }>96 \mathrm{~h} \\
{[\mathrm{n}, \%]}\end{array}$ & $59(90.77)$ & $47(21.17)$ & $<0.001$ & $36.61[14,42-108.45]$ & $<0.001$ & $40.51[13.65-120.25]$ \\
\hline \multirow{2}{*}{$\begin{array}{l}\text { CVC } \\
\text { Yes/No [n, \%] }\end{array}$} & 61 (93.85) & $129(58.11)$ & \multirow[t]{2}{*}{$<0.001$} & \multirow[t]{2}{*}{$10.99[3.85-42.83]$} & & \\
\hline & $4(6.15)$ & $93(41.89)$ & & & & \\
\hline \multirow{2}{*}{$\begin{array}{l}\text { Urinary catheter } \\
D \text { Yes/No [n, \%] }\end{array}$} & 61 (93.85) & $87(39.19)$ & \multirow[t]{2}{*}{$<0.001$} & \multirow[t]{2}{*}{$23.66[8.26-91.93]$} & & \\
\hline & $4(6.15)$ & $135(60.81)$ & & & & \\
\hline \multirow{2}{*}{$\begin{array}{l}\text { Hemodialysis } \\
\text { Yes/No [n, \%] }\end{array}$} & $7(10.77)$ & $8(3.60)$ & \multirow[t]{2}{*}{0.049} & \multirow[t]{2}{*}{$3.23[1.00-10.32]$} & & \\
\hline & $58(89.23)$ & $214(96.40)$ & & & & \\
\hline \multirow{2}{*}{$\begin{array}{l}\text { Tracheostomy } \\
\text { Yes/No [n, \%] }\end{array}$} & $41(63.08)$ & $28(|2.6|)$ & \multirow[t]{2}{*}{$<0.001$} & \multirow[t]{2}{*}{$12.35[6.18-24.91]$} & \multirow[t]{2}{*}{0.024} & \multirow[t]{2}{*}{$2.65[1.14-6.17$} \\
\hline & $23(35.38)$ & 194 (87.39) & & & & \\
\hline \multirow{2}{*}{$\begin{array}{l}\text { Gastrostomy } \\
\text { Yes/No [n, \%] }\end{array}$} & $2(3.08)$ & $7(3.15)$ & \multirow[t]{2}{*}{1.00} & \multirow[t]{2}{*}{$0.98[0.10-5.30]$} & & \\
\hline & $63(96.92)$ & $215(96.85)$ & & & & \\
\hline \multirow{2}{*}{$\begin{array}{l}\text { Vasoactive therapy } \\
\text { Yes/No [n, \%] }\end{array}$} & $46(70.77)$ & $43(19.37)$ & \multirow[t]{2}{*}{$<0.001$} & \multirow[t]{2}{*}{$10.08[5.14-19.92]$} & & \\
\hline & $19(29.23)$ & $179(80,63)$ & & & & \\
\hline \multirow{2}{*}{$\begin{array}{l}\text { Surgical wound } \\
\text { Yes/No [n, \%] }\end{array}$} & $52(80.00)$ & $189(85.14)$ & \multirow[t]{2}{*}{0.321} & $0.70[0.33-1.5 I]$ & & \\
\hline & $13(20.00)$ & $33(14.86)$ & & & & \\
\hline Pressure sores & $9(13.85)$ & $23(10.36)$ & 0.432 & $1.39[0.56-3.38]$ & & \\
\hline Yes/No [n, \%] & $56(86.15)$ & 199(89.64) & & & & \\
\hline Transfusions & $47(72.31)$ & $48(21.62)$ & $<0.001$ & $9.47[4.83-18.72]$ & & \\
\hline Yes/No [n, \%] & $18(27.69)$ & 174 (78.38) & & & & \\
\hline Nasogastric nutrition & 57 (87.69) & $53(23.87)$ & $<0.001$ & $22.72[9.68-55.26]$ & & \\
\hline Yes/No [n, \%] & $8(12.31)$ & $169(76.13)$ & & & & \\
\hline Immunosuppressive pathology & $5(7.69)$ & $12(5.4 I)$ & 0.549 & $1.46[0.39-4.66]$ & & \\
\hline Yes/No [n, \%] & $60(92.31)$ & $210(94.59)$ & & & & \\
\hline
\end{tabular}

(Continued) 
Table 5 (Continued).

\begin{tabular}{|l|l|l|l|l|l|l|}
\hline Variables & $\begin{array}{l}\text { CRP Group } \\
\text { nI=65 }\end{array}$ & $\begin{array}{l}\text { P-S Group } \\
\text { n2=222 }\end{array}$ & P & OR [95\% CI] & P & HR [95\% CI] \\
\cline { 1 - 5 } $\begin{array}{l}\text { Chemotherapy } \\
\text { Yes/No [n, \%] }\end{array}$ & $0(0.00)$ & $6(2.70)$ & 0.342 & $0.00[0.00-2.90]$ & & \\
\cline { 1 - 4 } $\begin{array}{l}\text { Radiotherapy } \\
\text { Yes/No [n, \%] }\end{array}$ & $05(100)$ & $216(97.30)$ & 1.00 & $0.00[0.00-133.20]$ & & \\
\cline { 1 - 4 } & $65(100)$ & $221(99.55)$ & & & \\
\hline
\end{tabular}

Notes: P-S group, subsample with carbapenem-sensitive Proteeae strains; CVC, central venous catheter; Nagelkerke $R^{2}=0.534$; Hosmer and Lemeshow test=0.929; $\mathrm{Cl}$, confidence interval.

Association between blaNDM and blaCTX-M was noticed in the case of 4CRP strains, $75 \%$ of these being P. stuartii. One $P$. stuartii strain in which no carbapenemase synthesis was identified showed the blaCTX-M gene. Most studies have shown the presence of the blaCTX-M gene in other Enterobacterales, such as $E$. coli and $K$. pneumoniae, responsible for healthcareassociated infections (HCAI). ${ }^{39-41}$

Studies on NDM-producing pathogens (Enterobacteriaceae, Acinetobacter, Pseudomonas) show that they frequently associate determinants of antimicrobial resistance such as AmpC, ESBL cephalosporinases, other carbapenemases (OXA-48, VIM and KPC), as well as factors that lead to resistance to other classes of antimicrobials. ${ }^{42-44}$ Therefore, most pathogens producing NDM-1 remain susceptible to only two bactericidal antibiotics (colistin and fosfomycin) and a single bacteriostatic one (tigecycline) ${ }^{44,45}$ Providencia species, however, have intrinsic resistance to colistin and tigecycline, so that the limited number of therapeutic options available will represent a real challenge for the clinician, especially when Proteeae strains are associated with other MDR-GNB strains, such as Acinetobacter species, with sensitivity preserved only to Colistin. ${ }^{46}$

There are studies in the literature that correlate the use and the length of colistin therapy with the increase in the prevalence of colonization and infections caused by strains having intrinsic resistance to colistin, such as Proteeae, Serratia marcescens, Pseudomonas mallei, and Burkholderia cepacia. ${ }^{15,46}$ Such increased colistin use was also noted in our hospital, ${ }^{17,47,48}$ which may explain the increased incidence of infections with Proteeae.

The main risk factors identified in our study for CRP infection were mechanical ventilation, especially if lasting $>96 \mathrm{~h}$, the presence of the urinary catheter, nasogastric nutrition and tracheostomy. Less frequently the CRP infections were associated with other procedures such as CVC insertion, vasoactive therapy and transfusions, which are part of medical care in ICU. After applying the logistic regression, three independent risk factors remained: mechanical ventilation lasting more than 96 hours, tracheostomy and days of antibiotherapy, noting that the lethality excess was $27.16 \%$.

In other studies performed in the same hospital, on other GNB, the risk factors for CRE infections were accounted for by the use of invasive medical devices, prolonged admission, antibiotic therapy, etc. ${ }^{46,47,49}$

Mariappan et al (2017) indicated as risk factors for CRE infections prolonged hospitalisation in ICU, mechanical ventilation, permanent medical devices, diabetes, use of several antibiotics, administration of carbapenems, focal infections or sepsis, as well as surgery. ${ }^{38}$

Clinical observations performed over the last 10 years in high-risk wards of our hospital have noted an upward trend and the persistence of infections with pathogens from the Proteeae tribe, frequently associated with bacterial MDR and low susceptibility to carbapenems. ${ }^{15,17,48}$ Because this phenomenon has been repeatedly reported, in the current study we wanted to highlight the behaviour of these strains according to their molecular pattern, risk factors identified and patients prognosis, all the more so as there are not enough data in the literature on this topic.

Our study has however some limitations: it was performed in a single tertiary medical unit, in a single university centre, with a specific program of surveillance and control of HCAI, which does not allow the generalization of the results.

\section{Conclusions}

There is a significant increase in the incidence of $\mathrm{CR}$ P. stuartii strains, isolated especially from bronchial aspirates and blood cultures of patients admitted to ICU and surgical departments, the MBL-blaNDM type being predominant. 
These strains presented various other resistance mechanisms, being often extremely difficult to treat and led to an excess of lethality of $27.16 \%$. Mechanical ventilation lasting for more than 96 hours, tracheostomy and prolonged antibiotic therapy were the three independent risk factors identified.

In view of this, a continuous optimization of the antibiotic stewardship policy should be considered, especially with regards to the use of colistin (but not only), in order to reduce the selective pressure exerted on bacteria with natural resistance to this antibiotic. Such an approach will also have a positive effect on reducing the mortality rate, the length of hospital stay and implicitly the costs.

\section{Abbreviations}

CLSI, Clinical Laboratory Standards Institute; bla, $\beta$ lactamase genes; CR, carbapenem resistant; CRE, carbapenem-resistant enterobacteria; CRP, carbapenemresistant Proteeae; CTX-M, cefotaximase-Munich; ESBL, extended spectrum beta-lactamase; GNB, Gramnegative bacilli; HCAI, healthcare associated infections; ICU, intensive care unit; IMP, imipenemase; KPC, Klebsiella pneumoniae carbapenemase; MBL, metallo- $\beta$ lactamases; MDR, multidrug resistant; MIC, minimum inhibitory concentration; NDM, New Delhi metallo-betalactamase; OXA, oxacillinase; PCR, polymerase chain reaction; SCJUPBT, Spitalul Clinic Judetean de Urgenta "Pius Brinzeu" Timisoara ("Pius Brinzeu" County Clinical Emergency Hospital Timișoara); TEM, Temoniera; VIM, Verona integron-encoded metallo- $\beta$ lactamase; CVC, central venous catheter.

\section{Disclosure}

The authors report no conflicts of interest in this work.

\section{References}

1. Adeolu M, Alnajar S, Naushad S, Gupta RS. Genome-based phylogeny and taxonomy of the 'Enterobacteriales': proposal for enterobacterales ord. nov. divided into the families Enterobacteriaceae, Erwiniaceae fam. nov., Pectobacteriaceae fam. nov., Yersiniaceae fam. nov., Hafniaceae fam. nov., Morgane. Int J Syst Evol Microbiol. 2016;66(12):5575-5599. doi:10.1099/ijsem.0.001485

2. O'Hara CM, Brenner FW, Miller JM. Classification, identification, and clinical significance of Proteus, Providencia, and Morganella. Clin Microbiol Rev. 2000;13(4):534-546. doi:10.1128/CMR.13.4.534

3. Iovleva A, Doi Y. Carbapenem-Resistant Enterobacteriaceae. Clin Lab Med. 2017;37(2). doi:10.1016/j.cll.2017.01.005

4. Codjoe F. Carbapenem Resistance: A Review. Med Sci. 2017. doi:10.3390/medsci6010001

5. Brenner FW. Who publishes list of bacteria for which new antibiotics are urgently needed. Saudi Med J. 2017.
6. Cabral AB, Maciel MAV, Barros JF, Antunes MM, Lopes ACS. Detection of blaKPC-2 in Proteus mirabilis in Brazil. Rev Soc Bras Med Trop. 2015;48(1):94-95. doi:10.1590/0037-8682-0152-2014

7. Tibbetts R, Frye JG, Marschall J, Warren D, Dunne W. Detection of KPC-2 in a clinical isolate of Proteus mirabilis and first reported description of carbapenemase resistance caused by a KPC $\beta$ lactamase in P. mirabilis. J Clin Microbiol. 2008;46(9):3080-3083. doi:10.1128/JCM.00979-08

8. Shiroto K, Ishii Y, Kimura S, et al. Metallo- $\beta$-lactamase IMP-1 in Providencia rettgeri from two different hospitals in Japan. $J$ Med Microbiol. 2005;54(11):1065-1070. doi:10.1099/jmm.0.46194-0

9. Sheng ZK, Li JJ, Sheng GP, Sheng JF, Li LJ. Emergence of Klebsiella pneumoniae carbapenemase-producing Proteus mirabilis in Hangzhou, China. Chin Med J. 2010. doi:10.3760/cma.j. issn.0366-6999.2010.18.014

10. Lange F, Pfennigwerth N, Gerigk S, et al. Dissemination of bla OXA-58 in Proteus mirabilis isolates from Germany. $J$ Antimicrob Chemother. 2017:dkw566. doi:10.1093/jac/dkw566

11. Potter RF, Wallace MA, McMullen AR, et al. blaIMP-27 on transferable plasmids in Proteus mirabilis and Providencia rettgeri. Clin Microbiol Infect. 2018;24(9):1019.e5-1019.e8. doi:10.1016/j.cmi.20 18.02.018

12. Lee HW, Kang HY, Shin KS, Kim J. Multidrug-resistant Providencia isolates carrying blaPER-1, blaVIM-2, and armA. J Microbiol. 2007.

13. Shibata N, Doi Y, Yamane K, et al. PCR Typing of Genetic Determinants for Metallo- $\beta$-Lactamases and Integrases Carried by Gram-Negative Bacteria Isolated in Japan, with Focus on the Class 3 Integron. J Clin Microbiol. 2003;41(12):5407-5413. doi:10.1128/ JCM.41.12.5407-5413.2003

14. Tsakris A, Poulou A, Themeli-Digalaki K, et al. Use of boronic acid disk tests to detect extended-spectrum $\beta$-lactamases in clinical isolates of KPC carbapenemase-possessing Enterobacteriaceae. $J$ Clin Microbiol. 2009;47(11):3420-3426. doi:10.1128/JCM.01314-09

15. Molnár S, Flonta MMM, Almaş A, et al. Dissemination of NDM-1 carbapenemase-producer Providencia stuartii strains in Romanian hospitals: a multicentre study. J Hosp Infect. 2019;103(2):165-169. doi:10.1016/j.jhin.2019.04.015

16. Székely E, Damjanova I, Jánvári L, et al. First description of blaNDM-1, blaOXA-48, blaOXA-181 producing Enterobacteriaceae strains in Romania. Int $J$ Med Microbiol. 2013;303(8):697-700. doi:10.1016/j.ijmm.2013.10.001

17. Axente C, Licker M, Moldovan R, et al. Antimicrobial consumption, costs and resistance patterns: A two year prospective study in a Romanian intensive care unit. BMC Infect Dis. 2017;17:358. doi:10.1186/s12879-017-2440-74

18. CLSI. Performance Standards for Antimicrobial Susceptibility Testing. 27th Ed. Wayne, PA: Clinical and Laboratory Standards Institute; 2017. doi:10.1039/C4DT01694G

19. Magiorakos AP, Srinivasan A, Carey RB, et al. Multidrug-resistant, extensively drug-resistant and pandrug-resistant bacteria: an international expert proposal for interim standard definitions for acquired resistance. Clin Microbiol Infect. 2012. doi:10.1111/j.1469-0691.2011.03570.x

20. Poirel L, Walsh TR, Cuvillier V, Nordmann P. Multiplex PCR for detection of acquired carbapenemase genes. Diagn Microbiol Infect Dis. 2011;70(1):119-123. doi:10.1016/j.diagmicrobio.2010.12.002

21. Akhi MT, Gholizadeh P, Ghotaslu R, et al. Fecal Carriage of ESBL types TEM, SHV, CTX Producing Genera Proteus, Morganella, Providencia in Patients of Iran. Ambient Sci. 2016;3(2):80-84. doi:10.21276/ambi.2016.03.2.ra06

22. Abdallah M, Balshi A. First literature review of carbapenem-resistant Providencia. New Microbes New Infect. 2018. doi:10.1016/j. nmni.2018.05.009

23. Olumuyiwa Olaitan A, Diene SM, Victor Assous M, Rolain JM. Genomic plasticity of multidrug-resistant NDM-1 positive clinical isolate of providencia rettgeri. Genome Biol Evol. 2016. doi:10.1093/gbe/evv195 
24. Barrios H, Garza-Ramos U, Reyna-Flores F, et al. Isolation of carbapenem-resistant NDM-1-positive Providencia rettgeri in Mexico. J Antimicrob Chemother. 2013;68(8):1934-1936. doi:10.10 93/jac/dkt124

25. Zurita J, Parra H, Gestal MC, McDermott J, Barba P. First case of NDM-1-producing Providencia rettgeri in Ecuador. $J$ Glob Antimicrob Resist. 2015;3(4):302-303. doi:10.1016/j.jgar.2015.07. 003

26. Pollett S, Miller S, Hindler J, Uslan D, Carvalho M, Humphries RM. Phenotypic and molecular characteristics of carbapenem-resistant Enterobacteriaceae in a health care system in Los Angeles, California, from 2011 to 2013. J Clin Microbiol. 2014;52 (11):4003-4009. doi:10.1128/JCM.01397-14

27. Manageiro V, Sampaio DA, Pereira P, et al. Draft genome sequence of the first NDM-1-producing Providencia stuartii strain isolated in Portugal. Genome Announc. 2015;3(5). doi:10.1128/genomeA.01077-15

28. Mc Gann P, Hang J, Clifford RJ, et al. Complete sequence of a novel 178-kilobase plasmid carrying bla NDM-1 in a Providencia stuartii strain isolated in Afghanistan. Antimicrob Agents Chemother 2012;56(4):1673-1679. doi:10.1128/AAC.05604-11

29. Galani L, Galani I, Souli M, et al. Nosocomial dissemination of Providencia stuartii isolates producing extended spectrum $\beta$ lactamases VEB-1 and SHV-5, metallo- $\beta$-lactamase VIM-1, and RNA methylase RmtB. $J$ Glob Antimicrob Resist. 2013;1 (2):115-116. doi:10.1016/j.jgar.2013.03.006

30. Oikonomou O, Liakopoulos A, Phee LM, Betts J, Mevius D, Wareham DW. Providencia stuartii isolates from Greece co-carriage of cephalosporin (bla SHV-5 bla VEB-1), carbapenem (bla VIM-1), and aminoglycoside ( $\mathrm{rmtB})$ resistance determinants by a multidrug-resistant outbreak clone. Microb Drug Resist. 2016;22 (5):379-386. doi:10.1089/mdr.2015.0215

31. Douka E, Perivolioti E, Kraniotaki E, et al. Emergence of a pandrug-resistant VIM-1-producing Providencia stuartii clonal strain causing an outbreak in a Greek intensive care unit. Int J Antimicrob Agents. 2015;45(5):533-536. doi:10.1016/j.ijantimicag.2014.12.030

32. Robin F, Aggoune-Khinache N, Delmas J, Naim M, Novel BR. VIM metallo- $\beta$-lactamase variant from clinical isolates of Enterobacteriaceae from Algeria. Antimicrob Agents Chemother 2010;54(1):466-470. doi:10.1128/AAC.00017-09

33. Bocanegra-Ibarias P, Garza-González E, Morfín-Otero R, et al. Molecular and microbiological report of a hospital outbreak of NDM-1-carrying Enterobacteriaceae in Mexico. PLoS One. 2017;12 (6):e0179651. doi:10.1371/journal.pone.0179651

34. Vourli S, Tsorlini H, Katsifa H, et al. Emergence of Proteus mirabilis carrying the blaVIM-1 metallo- $\beta$-lactamase gene. Clin Microbiol Infect. 2006;12(7):691-694. doi:10.1111/j.1469-0691.2006.01489.x

35. Bontron S, Poirel L, Kieffer $\mathrm{N}$, et al. Increased Resistance to Carbapenems in Proteus mirabilis Mediated by Amplification of the blaVIM-1 -Carrying and IS 26 -Associated Class 1 Integron. Microb Drug Resist. 2019;25(5):663-667. doi:10.1089/mdr.2018.0365

36. Poirel L, Schrenzel J, Cherkaoui A, Bernabeu S, Renzi G, Nordmann P. Molecular analysis of NDM-1-producing enterobacterial isolates from Geneva, Switzerland. J Antimicrob Chemother. 2011;66(8):1730-1733. doi:10.1093/jac/dkr174

Infection and Drug Resistance

\section{Publish your work in this journal}

Infection and Drug Resistance is an international, peer-reviewed openaccess journal that focuses on the optimal treatment of infection (bacterial, fungal and viral) and the development and institution of preventive strategies to minimize the development and spread of resistance. The journal is specifically concerned with the epidemiology of
37. Yang J-H, Sheng W-H, Hsueh P-R. Antimicrobial susceptibility and distribution of extended-spectrum $\beta$-lactamases, AmpC $\beta$-lactamases, and carbapenemases among Proteus, Providencia, and Morganella isolated from global hospitalized patients with intra-abdominal and urinary tract infections. J Glob Antimicrob Resist. 2020. doi:10.1016/ j.jgar.2020.04.011

38. Mariappan S, Sekar U, Carbapenemase-producing Enterobacteriaceae KA. Risk factors for infection and impact of resistance on outcomes. Int J Appl Basic Med Res. 2017;7(1):32. doi:10.4103/2229-516x.198520

39. Licker M, Anghel A, Moldovan R, et al. Genotype-phenotype correlation in multiresistant Escherichia coli and Klebsiella pneumoniae strains isolated in Western Romania. Eur Rev Med Pharmacol Sci. 2015;19(10): 1888-1894.

40. Abrar S, Vajeeha A, Ul-Ain N, Riaz S. Distribution of CTX-M group I and group III $\beta$-lactamases produced by Escherichia coli and Klebsiella pneumoniae in Lahore, Pakistan. Microb Pathog. 2017. doi:10.1016/j.micpath.2016.12.004

41. Wang G, Huang T, Surendraiah PKM, et al. CTX-M $\beta$ Lactamase-producing Klebsiella pneumoniae in Suburban New York City, New York, USA. Emerg Infect Dis. 2013;19 (11):1803-1810. doi:10.3201/eid1911.121470

42. Bonnin RA, Poirel L, Licker M, Nordmann P. Genetic diversity of carbapenem-hydrolysing $\beta$-lactamases in Acinetobacter baumannii from Romanian hospitals. Clin Microbiol Infect. 2011;17 (10):1524-1528. doi:10.1111/j.1469-0691.2011.03622.x

43. Castanheira M, Deshpande LM, Mathai D, Bell JM, Jones RN, Mendes RE. Early Dissemination of NDM-1- and OXA-181Producing Enterobacteriaceae in Indian Hospitals: report from the SENTRY Antimicrobial Surveillance Program, 2006-2007. Antimicrob Agents Chemother. 2011;55(3):1274-1278. doi:10.1128/ AAC.01497-10

44. Falagas ME, Karageorgopoulos DE, Nordmann P. Therapeutic options for infections Enterobacteriaceae producing carbapenem-hydrolyzing enzymes. Future Microbiol. 2011;6(6):653-666. doi:10.2217/fmb.11.49

45. Dortet L, Poirel L, Nordmann P. Worldwide dissemination of the NDM-Type carbapenemases in Gram-negative bacteria. Biomed Res Int. 2014;2014:1-12. doi:10.1155/2014/249856

46. Muntean D, Licker M, Horhat F, et al. Extensively drug-resistant Acinetobacter baumannii and Proteeae association in a Romanian intensive care unit: risk factors for acquisition. Infect Drug Resist. 2018;11:2187-2197. doi:10.2147/IDR.S1712886

47. Baditoiu L, Axente C, Lungeanu D, et al. Intensive care antibiotic consumption and resistance patterns: a cross-correlation analysis. Ann Clin Microbiol Antimicrob. 2017;16:71. doi:10.1186/s12941-0170251-87

48. Muntean D, Horhat FG, Badiţoiu L, et al. Multidrug-resistant gramnegative bacilli: a retrospective study of trends in a tertiary healthcare unit. Medicina. 2018;54(6):92. doi:10.3390/MEDICINA54060092

49. Licker M, Sorescu T, Rus M, et al. Extensively drug-resistant Myroides odoratimimus - a case series of urinary tract infections in immunocompromised patients. Infect Drug Resist. 2018;11:743-749. doi:10.2147/IDR.S161069 antibiotic resistance and the mechanisms of resistance development and diffusion in both hospitals and the community. The manuscript management system is completely online and includes a very quick and fair peerreview system, which is all easy to use. Visit http://www.dovepress.com/ testimonials.php to read real quotes from published authors. 